\title{
Review of Traffic Signal Control based on Fuzzy Logic
}

\author{
Nidhi Sharma \\ M.Tech Scholar, \\ Department of Computer and \\ Science engineering, \\ Ajay Kumar Garg Engineering College, \\ Ghaziabad, India
}

\author{
Shashank Sahu \\ Associate Professor, Department of Computer and \\ Science Engineering \\ Ajay Kumar Garg Engineering College, \\ Ghaziabad,India
}

\begin{abstract}
In the present time the number of private vehicles is increasing rapidly. As result the traffic congestion is growing and becoming a huge problem in big cities. Due to traffic congestion many huge problems are occurring like pollution, wastage of time, money, accidents etc. Traffic congestion occurred very frequently in the present time which affect the daily life and introduce so many problems and challenges. To solve all these problems traffic signal control is a best solution. Fuzzy set theory have been widely applied to many applications and it can easily deal with uncertainty, complexity and imprecision in many systems. The problems in traffic engineering are mainly characterized by the imprecise, ambiguous and uncertain parameters. Due to such type of parameters fuzzy logic method is best suitable approach for traffic signal control. The performance of fuzzy logic based controller is better for two one way street based on extension of green light time. Later fuzzy logic controller was developed for two way intersection without turns, single intersection with all movements, multiple intersection etc. Better performance of fuzzy logic based traffic signal control in comparison of traditional traffic signal control increase the research in this field. Fuzzy logic approach will definitely help in transportation management system in future.
\end{abstract}

\section{Keywords}

Traffic signal control, fuzzy logic

\section{INTRODUCTION}

Zadeh [1] in 1965 proposed the concept of fuzzy logic which stems for fuzzy sets. Fuzzy set theory has been applied on many applications for dealing with uncertainty, imprecision and complexity. However the real world is uncertain and imprecise, fuzzy set theory is best suitable for dealing with real world problems by developing the precise conclusions The main and basic elements of fuzzy set theory are knowledgebase in which the working of fuzzy system is stored in the form of if-then rules, inputs in crisp manner, outputs which are also in crisp manner and an inference system which compares the input with the knowledge stored in knowledge based after that the output is determined.

Based on the study fuzzy set theory can be applied in many application areas which are as follow:

1. Processes where one need to mix many type of information such as quantitative information, qualitative information expressed in linguistic terms and measurements.

2. Processes where decision making and human reasoning are required.
3. The systems which are very complex to understand. And the computed solution with all known variables is impossible.

4. Problems which are characterized by natural language [2].

5. Systems which required the capability of human common-sense reasoning and recognizing capability.

According to Hoogendoorn et al. [3] the benefits of fuzzy logic approach are as follow:

- Fuzzy systems are able to deal with words and sentences expressed in natural language. Hence communication of system with the user is improved.

- Fuzzy systems can easily handle the quantitative and qualitative information such as human experiences in linguistic terms.

- Fuzzy control provides transparent and flexible control structure.

- Fuzzy systems can easily process the complex and decision making processes.

- Fuzzy logic system is good for dealing with nonlinear input and output relationship.

Some drawbacks of fuzzy logic approach are as follow:

- $\quad$ To find out the relationship among the parameters describing membership functions is a problem because the effect of different parameters is local.

- For stability analysis of fuzzy controller system no methodology is developed yet.

- Mostly the fuzzy logic based findings which provide improved performances are simulation result based

The following paper is divided in four sections. First section explains traffic signal control problem. Second section provides application of fuzzy logic in traffic engineering. The third section explains the progression of fuzzy logic based traffic signal control. And finally fourth section concludes the paper.

\section{TRAFFIC SIGNAL CONTROL}

In the present time the traffic light system changes the light after a fixed given interval. An intelligent traffic signal senses the presence and absence of vehicles and changes its timings of signals according to that. This system also aims to have efficient and safe traffic flow control with reduced waiting time at signal intersection and heavy traffic jams. The traffic congestion can be reduced by increasing the time of green signal on congested road and decreasing the time of red signal on non congested road. 
TSC is a very important and effective traffic control method safe and quick travel. TSC systems have undergone for continuous improvement. TSC methods are passed through three stages. First is pre-time controlled, second is traffic responsive control and last one is intelligent control. The real time traffic measurement is commonly available by the use of many advanced sensing and communication technologies. For stable and regular traffic flow fixed signal time control method is suitable. For the traffic study of network TRANSYT [4] is a good example. For getting the real time traffic information from the environment sensing technology is used. Any traffic control strategy can be made easily with the help of current real time traffic data. Some adaptive traffic systems are Split cycle offset optimization technique (SCOOT) [5], Sydeny coordinated adaptive traffic system (SCATS), Itly's urban traffic optimization by integrated optimization of traffic (SPOT). There are so many factors which affect the traffic control system like traffic infrastructure, travelers, number of vehicles, weather conditions, etc. Each and every factor has its own properties, which make the traffic system large and complicated, which create many problems and challenges for researchers and engineers.

\section{APPLICATION OF FUZZY LOGIC IN TRAFFIC ENGINEERING}

In traffic system some phenomena like route choice behavior, driving behavior, or traffic management result from subjective decisions. The input data which is used to take these decisions are travel cost, travel time, distance and velocity of vehicles, congestion duration, waiting time, etc. Some of these input data are crisp while others are ambiguous and described by the linguistic variables. Hence in traffic engineering large range of problems are defined by variables that are uncertain, vague, subjective, and imperfect. The traffic engineering research field has a scarcity of analytical tools which can easily handle the ambiguity in human perception and decision making process. Fuzzy logic can be applied to deal with such type of problems in the research field of traffic engineering. Some applications are incident detection, congestion detection modeling route choice behavior, management of parking and traffic control.

First time "Pappis and Mamdani" [6] presented the paper in which fuzzy logic is used for solving practical traffic and transportation problem. They presented a controller based on fuzzy logic for a two one way streets based single intersection and results are compared with vehicle actuated controller. The controller results in a good performance. Teodorovic [7] present a fuzzy logic controller based mathematical approach to model complex , ambiguous, uncertain, and imprecise traffic and transportation processes.

Applications of fuzzy logic in different areas of traffic engineering and transportation management are categorized as follow:

1. Some of fuzzy logic based applications in traffic engineering are found in real life. Most of the applications are in developing phase and are applied in a simulated environment which is unrealistic and oversimplified.

2. The results of fuzzy logic applications are satisfactory whether in real life environment or in simulated environment.

3. The results show that the fuzzy set theory can easily be able to deal with uncertainty, vagueness, and ambiguity in traffic engineering such as car following behavior and route choice.

4. Fuzzy logic fuses the data from many different resources which increases the accuracy of the predictions and estimations.

5. Generally the fuzzy approaches which have been applied in researches do not efficiently use the fuzzy methodologies.

\section{TRAFFIC SIGNAL CONTROL BASED ON FUZZY LOGIC}

Traffic signal controls can be pre-timed control or actuated control or adaptive control. The pre-timed controller uses the predetermined signal timings collected from older traffic pattern and repeat it. Actuated controllers detect the moving and paused traffic on all lanes towards intersection and calculate the signal phase duration. Adaptive controllers consider the whole intersection and adjust signal phase and timings in response to real time traffic.

In adaptive control, artificial intelligence techniques are playing an important role by adjusting green time, cycle length, red time, and phase sequence. The fuzzy logic approach is used in adaptive traffic signal control to explore expert's knowledge. Fuzzy describes the uncertainty in the environment. It is a contrast of crisp logic in which there are only two values true or false. Fuzzy logic shows certain degree of true or false. Fuzzy theory is first proposed by Zadeh [8]. One of the best features of fuzzy system is the ability to include an expert's knowledge in their design. Fuzzy theory has been applied in many fields of traffic control system to solve many problems. It has been applied to control traffic signal, vehicles, lifts, home appliances, etc.

The fuzzy logic based system might be the first successful attempt to implement intelligence in traffic control system. The fuzzy system translates the input to the output of the system. In system domain the linguistic values are divided in fuzzy sets, for example we can define traffic flow as low, high and medium. Membership functions show the degree of dependency to each fuzzy set. Value of input in fuzzy system may belong to more than on fuzzy set. The association of numerical values to fuzzy set is called fuzzification and the reverse process is called defuzzification. The main logic of the fuzzy system is defined by if-then rules in the fuzzy inference.

The fuzzy system is a vague reasoning system so it can be easily applied to many application fields. A priori expert knowledge of objects can be easily reflected in well designed fuzzy rules so there is no need of mathematical models of objects. Fuzzy system is very flexible and its modification is not so difficult. To achieve better results the membership function parameters and their quantified values, system operators, fuzzy rules, defuzzification methods and other parameters can be changed for achieving good results.

Pappis and Mamdani firstly presented a paper in which a fuzzy logic controller of two intersection one-way streets was implemented. The results obtained from controller are tabulated and compared with the conventional effective vehicle actuated controller. The performance crieterion vehicle's average delay shows that the performance of fuzzy logic controller is better. Yousaf [9] proposed an autonomus traffic light control system based on multiagent approach using fuzzy control. The proposed system can minimize the vehicle waiting time using fuzzy logic controller under the 
situations that normally occurred during emergency. This system was implemented on two traffic junctions.

$\mathrm{Wu}$ Wei and Y. Zhang et al. [10] developed adaptive traffic signal controller based on fuzzy logic for four way intersection approach. The controller makes adjustment to signal timings according to observed changes. The extension or termination of green light time and sequences of phases can be determined using three levels model of fuzzy control. Multiobjective genetic algorithm was used to find a set of optimal parameters for fuzzy logic controller. Wu Wei and Yi Zhang et al. [11] developed a fuzzy logic based traffic signal controller for an isolated four approaches intersection. They also use multi objective genetic algorithm (MOGA) to find a set of optimal parameters for fuzzy controller.

Qiao et al. [12] developed a two stage fuzzy logic control method for an isolated signalized intersection, where both traffic efficiency and fairness were considered simultaneously. The simulation demonstrated that this system outperformed the vehicle actuated controlling all performance indices.

Gokulan B. P and D. Srinivasan [21] adopted a distributed multi-agent based approach i.e. a traffic signal control was developed based on geometric fuzzy multi-agent system approach. This system used a geometric type-2 fuzzy inference system which is capable of handling uncertainty found in inputs and rule base of traffic signal controller. The comparison of this controller was performed against the existing traffic control algorithms hierarchical multiagent system and green link determining.

P.G. Balaji and D.Srinivasan [22] also proposed a type-2 fuzzy decision system based multi-agent architecture. The green phase time was calculated using the weights calculated for the flow input and data collected from road network. Alper [23] built a real time traffic simulator utilizing an adaptive fuzzy inference mechanism by tuning fuzzy parameters. In this simulator the time duration of light is changed according to waiting vehicles behind green and red light at crossroad. The results are compared with Webster road research technical paper and HCM.

Abel Nassir [24] proposed a traffic system based on fuzzy logic for a two way intersection and which is able to change signal timings according to situation level. This system provides good performance in terms of total moving time, average waiting time, and queue length.

Niittymaki [27] applied fuzzy logic for pedestrian crossing signal. This signal control was tested against two types of demand actuated control: one that used the traditional green time extension and other that used the modified extension rules. Evolution criteria were delay to pedestrian and vehicles and percentage of vehicles stopped. The summary for evolution of traffic signal control based on fuzzy logic is illustrated in Table 1. 


\begin{tabular}{|l|l}
\hline Authors & Area of contribution \\
\hline
\end{tabular}

J. Niittymaki (2001) They presented a paper in which fuzzy rule base formulation is done for separate single intersection. [10]

Wu Wei and Yi Zhang et al.(2001) [11]

F. Qiao et al.(2002) [12]

Chih-Hsun and JenChao(2002) [13]

Isiakki Konsen(2003) [14]

Jarkko Niittymaki and Esko Turunen(2003) [15]

Zhang, Li, and Prevedouros(2004) [16]

ANA L. C. BAZZAN(2005) [17]

Lilin Zang et al.(2006 )[18]

Loukas Diitriou et al.(2007) [19]

Mohammad Hossaien et al.(2009) [20]

Gokulan, B. P., \& Srinivasan,

D.(2010)[21]

P. G. Balaji and D. Srinivasan(2010) [22]

Alper(2011) [23]

Abdel Nasser H. Zaied and Woroud Al Othman(2011) [24]

Mohammad A. Taha (2012) [25]

Yunrui Bi et al(2014) [26]
They developed a fuzzy logic based traffic signal controller for an isolated four approaches intersection. They also use multi objective genetic algorithm (MOGA) to find a set of optimal parameters for fuzzy controller.

They developed a fuzzy logic based delay estimation system which can treat both technical and nontechnical factors. They simulate the signal controller for over congested Intersections using fuzzy logic. The controller extends or terminates the green phase for given real time traffic information.

They implemented traffic junction signal controller based on fuzzy logic which accounts number of continuous junctions, number of lanes, length of vehicles and length of roads. It has following properties: different input variables, lesser inference frequency, lesser rules and connecting each junction with others.

Presented a traffic signal control system which was based on multi-agent control scheme, real time traffic simulation, and fuzzy inference system.

They represented a traffic signal control which was based on fuzzy logic based reasoning. In this fuzzy reasoning was tied to many valued logic frame work.

They developed a fuzzy logic controller which worked as an experienced human traffic controller. According to real time traffic information it extended or terminated the green phase based on fuzzy rule base.

Presented an approach in which traffic signal agents were used. For coordination between the agents a distributed approach was used.

They presented a signal control algorithm for traffic signal control which was based on fuzzy logic by optimizing the phase's order of traffic signal.

They developed a hybrid approach based on fuzzy rule base for modeling the urban traffic flow and short term forecasting of urban traffic flow.

They presented fuzzy signal control system which contained fuzzy phase selector for selecting the signal phase and fuzzy green phase extender for extending the green phase.

They developed urban traffic signal control using distributed geometric fuzzy multi-agent which is based on a geometric type-2 fuzzy inference system.

They presented a multi agent system which was using the type- 2 fuzzy decision module for traffic signal control in a complex urban road network.

They developed traffic simulator based on real time traffic information using fuzzy inference mechanism by tuning fuzzy parameters.

They proposed a fuzzy logic based traffic system for single intersection which considers the two way intersection.

They presented a system for traffic simulation based on fuzzy logic.

They proposed type-2 fuzzy logic control method using multi-agent approach for multiple intersection traffic signal control optimized by differential evolution.

\section{CONCLUSION}

In the present time for urban traffic management traffic light plays an important role. So there is a need to improve the traffic signal control for traffic management and better traffic flow leading to greener environment. The parameters and variables in traffic engineering are based on subjectivity, imprecision, and ambiguity. Fuzzy approach is adequate in dealing with ambiguity, subjectivity, indetermination, and uncertainty present in traffic engineering. The methods 
provided by fuzzy logic take care of safety, efficiency and environmental objectives in developing efficient and effective traffic signal control. The values of initial parameters in traffic signal control based on fuzzy logic are adjustable and are also using different inference methods for exploiting better performance. The fuzzy logic approach in traffic signal control shows better performance. When the performance of fuzzy approach based traffic signal control is compared with the traditional traffic signal control, it shows better results. The study of fuzzy logic approach in traffic signal control shows that fuzzy logic based traffic signal control can easily handle the high congestion problem and uneven traffic flow on single intersection as well as on multi-intersection on road network

\section{REFERENCES}

[1] Zadeh, Lotfi A. "Fuzzy sets." Information and control 8.3 (1965): 338-353.

[2] Zadeh, Lotfi A. "The concept of a linguistic variable and its application to approximate reasoning-I." Information sciences 8.3 (1975): 199-249.

[3] Hoogendoorn, Serge Paul, G. Copinga, and U. Kaymak. Perspectives of fuzzy logic in traffic engineering: main document. Ministry of Transport, Public Works and Water Management, 1998.

[4] Robertson, Dennis I. "TRANSYT: a traffic network study tool." (1969).

[5] Hunt, P. B., D. I. Robertson, R. D. Bretherton, and R. I. Winton. SCOOT-a traffic responsive method of coordinating signals. No. LR 1014 Monograph. 1981

[6] Pappis, C. P., \& Mamdani, E. H. (1977). A fuzzy logic controller for a trafc junction. Systems, Man and Cybernetics, IEEE Transactions on, 7(10), 707-717.

[7] Teodorović, Dušan. "Fuzzy logic systems for transportation engineering: the state of the art." Transportation Research Part A: Policy and Practice 33.5 (1999): 337-364.

[8] Zadeh, Lotfi Asker. "Fuzzy sets as a basis for a theory of possibility." Fuzzy sets and systems 1, no. 1 (1978): 328.

[9] Saeed, Yousaf, M. Saleem Khan, Khalil Ahmed, and A. Salam Mubashar. "A multi-agent based autonomous traffic lights control system using fuzzy control." International Journal of Scientific \& Engineering Research 2, no. 6 (2011): 1.

[10] Niittymäki, Jarkko. "General fuzzy rule base for isolated traffic signal control-rule formulation." Transportation Planning and Technology 24.3 (2001): 227-247.

[11] Wei, Wu, et al. "Traffic signal control using fuzzy logic and MOGA."Systems, Man, and Cybernetics, 2001 IEEE International Conference on. Vol. 2. IEEE, 2001.

[12] Qiao, Fengxiang, et al. "Fuzzy logic based intersection delay estimation."Mathematical and computer modelling 36.11 (2002): 1425-1434.

[13] Chou, Chih-Hsun, and Jen-Chao Teng. "A fuzzy logic controller for traffic junction signals." Information Sciences 143.1 (2002): 73-97.
[14] Kosonen, Iisakki. "Multi-agent fuzzy signal control based on real-time simulation." Transportation Research Part C: Emerging Technologies 11.5 (2003): 389-403.

[15] Niittymäki, Jarkko, and Esko Turunen. "Traffic signal control on similarity logic reasoning." Fuzzy Sets and Systems 133.1 (2003): 109-131.

[16] Zhang, Lin, Honglong Li, and Panos D. Prevedouros. "Signal control for oversaturated intersections using fuzzy logic." Transportation Research Board Annual Meeting, Washington DC, USA. 2005.

[17] Bazzan, Ana LC. "A distributed approach for coordination of traffic signal agents." Autonomous Agents and Multi-Agent Systems 10.1 (2005): 131-164.

[18] Zang, Lilin, Lei Jia, and Zhongqin Lin. "Research of traffic signal control and simulation based on fuzzy logic." Journal of Highway and Transportation Research and Development (English Edition) 1.1 (2006): 96-99.

[19] Dimitriou, Loukas, Theodore Tsekeris, and Antony Stathopoulos. "Adaptive hybrid fuzzy rule-based system approach for modeling and predicting urban traffic flow." Transportation Research Part C: Emerging Technologies 16.5 (2008): 554-573.

[20] Zarandi, Mohammad Hossein Fazel, and Shabnam Rezapour. "A fuzzy signal controller for isolated intersections." Journal of Uncertain Systems 3.3 (2009): 174-182.

[21] Gokulan, Balaji Parasumanna, and Dipti Srinivasan. "Distributed geometric fuzzy multiagent urban traffic signal control." Intelligent Transportation Systems, IEEE Transactions on 11.3 (2010): 714-727.

[22] Balaji, P. G., and Dipti Srinivasan. "Multi-agent system in urban traffic signal control." Computational Intelligence Magazine, IEEE 5.4 (2010): 43-51.

[23] Aksaç, Alper, Erkam Uzun, and Tansel Özyer. "A real time traffic simulator utilizing an adaptive fuzzy inference mechanism by tuning fuzzy parameters." Applied Intelligence 36, no. 3 (2012): 698-720.

[24] Zaied, Abdel Nasser H., and Woroud Al Othman. "Development of a fuzzy logic traffic system for isolated signalized intersections in the State of Kuwait." Expert Systems with Applications 38.8 (2011): 9434-9441.

[25] Taha, Mohammad A., and Laheeb Ibrahim. "Traffic simulation system based on fuzzy logic." Procedia Computer Science 12 (2012): 356-360.

[26] Bi , Yunrui, et al. "Type-2 fuzzy multi-intersection traffic signal control with differential evolution optimization." Expert Systems with Applications 41.16 (2014): 7338-7349.

[27] Niittymaki, Jarkko, and Shinya Kikuchi. "Application of fuzzy logic to the control of a pedestrian crossing signal." Transportation Research Record: Journal of the Transportation Research Board 1651 (1998): 30-38. 